Cahiers de recherches médiévales

\title{
L'effusion de sang dans les procès et les traités concernant Jeanne d'Arc (1430-1456)
}

Françoise Michaud-Fréjaville

\section{OpenEdition}

Édition électronique

URL : https://journals.openedition.org/crm/731

DOI : $10.4000 / \mathrm{crm} .731$

ISSN : 1955-2424

Éditeur

Honoré Champion

Édition imprimée

Date de publication : 1 juin 2005

Pagination : 179-187

ISSN : 1272-9752

\section{Référence électronique}

Françoise Michaud-Fréjaville, «L'effusion de sang dans les procès et les traités concernant Jeanne d'Arc (1430-1456) », Cahiers de recherches médiévales [En ligne], 12 spécial | 2005, mis en ligne le 28 juin 2008, consulté le 15 décembre 2022. URL : http://journals.openedition.org/crm/731 ; DOI :

https://doi.org/10.4000/crm.731 


\title{
rig
}

\author{
$-15-$ \\ L'effusion de sang dans les procès \\ et les traités concernant Jeanne d'Arc (1430-1456)
}

Les 27 et 28 mars 1431 (n. st.), Jean Estivet, le promoteur du procès de Rouen, exposa devant Jeanne d'Arc soixante-dix articles rappelant les réponses de la jeune fille aux diverses accusations formulées contre elle au cours des séances du procès. Le $25^{\mathrm{e}}$ article précisait ${ }^{1}$ :

Ladite Jeanne, usurpant l'office des anges, a dit et affirmé qu'elle a été et qu'elle est envoyée de par Dieu, même pour des actes qui tendent entièrement à la voie de fait et à l'effusion de sang, ce qui est absolument étranger à la sainteté et pour tout esprit pieux horrible et abominable.

Estivet mélange, selon le procédé dont il usa tout au long de son libelle, ses propres commentaires, «Jeanne usurpant l'office des anges, ce qui est étranger à la sainteté etc. » avec des fragments des déclarations de la jeune fille. À cet article elle rétorqua seulement qu'elle avait toujours voulu faire la paix : primo requirebat quod fieret pax ${ }^{2}$, avant d'être prête à combattre et casu que non fierat pax, parata erat pugnare. Et ce sont les greffiers qui notèrent en sus qu'elle avait confessé être venue de par Dieu le 24 février précédent ${ }^{3}$. Jeanne ne répondit en aucune manière directement à l'accusation d'avoir provoqué, au nom de Dieu, l'effusion de sang.

L'essai qui suit tente de discerner les attitudes des accusateurs puis des défenseurs de la Pucelle, confrontés avec l'inouï: une femme guerrière, dans une guerre sans dentelles, peut-elle survenir au nom de Dieu? L'embarras des uns et des autres est certain. Peut-on mener un combat militaire sans verser le sang? Doit-on magnifier les actions des femmes fortes au détriment des valeurs combatives viriles «naturelles»? Quand le sang versé est-il impur et quand peut-il sans offenser Dieu abreuver les sillons?

En vérité, et je dois le dire d'entrée de jeu, pas plus les juges de Rouen que ceux qui annulèrent son procès en 1456 n'ont résolu le dilemme de cette intrusion féminine, problème particulièrement ardu pour la civilisation de l'époque. Ils s'en sont tirés au mieux par le détournement du sujet, au pire par le silence. Les accusateurs ont insisté sur la cruauté et le détournement des valeurs viriles, les défenseurs, récusant l'accusation, l'ont retournée sur les juges, ou ont choisi de placer Jeanne

${ }^{1}$ Pierre Tisset et Yvonne Lanhers, Procès de condamnation de Jeanne d'Arc, Paris, Klincksieck, 1960-1971 (Société d'Histoire de France), t. I, p. 223 : Dicta Johanna officium angelorum usurpando, se dixit et assuerit et esse missam ex parte Dei, eciam ad ea que ad viam facti et sanguinis humani effusionem omnino tendunt; quod sanctitati penitus alienum est, et omni pie menti horrendum est et abominablilis. La référence à cet ouvrage sera ensuite : Procès de condamnation. ${ }^{2} I d$.

${ }^{3}$ Ibid., t. I, p. 56 [session du samedi 24 février] : dixit ulterius quod venit ex parte Dei; ibid., t. I, p. 57 : vox dixit ei quod respondere audacter et quod Deus juvaret eam.

Cahiers de Recherches Médiévales (XII -XV s.), 12spé, 2005 
d'Arc dans le groupe des héroïnes bibliques auquel en vérité, elle est difficilement intégrable.

\section{L'accusation : la cruauté}

Le 23 mai 1431, au cours de son admonestation, Pierre Morice apostropha Jeanne :

«Tu t'es vantée de faire tuer tous ceux qui n'obéissaient pas et qu'aux coups on verrait qui aurait meilleur droit de Dieu au ciel [...] Tu es traîtresse, fourbe, souhaitant cruellement l'effusion de sang humain, incitant à la tyrannie $»^{4}$.

Ces accusations, reprises du sixième article des conclusions de l'Université de Paris ${ }^{5}$ et que Jeanne aurait reconnues en ces mêmes termes lors de son abjuration $^{6}$, ne sont qu'une partie des reproches adressés à la jeune fille, elles sont toujours liées à la cruauté.

La cruauté de Jeanne se serait manifestée à chaque fois qu'elle a pris les armes car elle n'a épargné ni les autres ni les siens. Par deux fois, au moins, elle aurait dévoilé au grand jour cette cruauté qui prend plaisir à la souffrance. La première fois lors de l'exécution de Franquet d'Arras à Lagny en mai-juin $1430^{7}$, ensuite quand elle souhaita que Guichart Bouren le capitaine de Soissons qui refusa d'ouvrir sa ville fût écartelé, plus exactement coupé en quatre ${ }^{8}$. En écrivant dans sa lettre aux Anglais du 22 mars 1429, envoyée de Poitiers pendant le siège d'Orléans, que si les Anglais ne voulaient pas quitter le royaume de France elle les ferait "tous occire », elle montrait sans ambages sa résolution homicide envers ses ennemis. Mais Jeanne n'épargnait pas plus ses amis : à Orléans il y a eu plus de cent blessés ${ }^{10}$; à Paris, elle a permis que de nombreux hommes de son armée soient blessés, elle-même le fut

${ }^{4}$ Ibid., t. I, p. 377 : Desirans crudeliter effusionem sanguinis umani, sediciosa, provocans tirannicidem. La traduction par «assoiffée de sang humain» (ibid., t. II, p. 328), fournit une image qui ne se trouve pas dans le texte à cet emplacement mais dans le chapeau des articles du promoteur ! L'expression : "on verrait qui aurait meilleur droit de Dieu au ciel» était extraite de la lettre de Jeanne d'Arc aux Anglais du 22 mars 1429, citée intégralement à l'article XXII du libelle d'Estivet.

${ }^{5} I b i d .$, t. I, p. 362.

${ }^{6}$ «En désirant crueusement effusion de sang humain ».

${ }^{7}$ Ibid., t. I, p. 151. L'exécution du capitaine de routiers bourguignon Franquet est un des sujets de controverse de l'histoire johannique. Jean Chartier, chroniqueur partisan de Charles, qui parle de la victoire de Jeanne et des Français sur les Anglais à Lagny, en mai 1430, ne cite pas particulièrement cet épisode : "finablement furent tous iceulx Anglois mors et prins» (J. Chartier, Chronique de Charles VII, éd. Vallet de Viriville, Paris, 1858, t. I, p. 121). Chastellain, chroniqueur bourguignon, dit au contraire : «Il [Franquet] fut décapité par la crudelté de cete femme qui désiroit sa mort $»$.

${ }^{8}$ Procès de condamnation, t. I, p. 208, séance du 3 mars 1431.

${ }^{9}$ Ibid., t. I, 224.

${ }^{10}$ Ibid., t. I, p. 195, session du samedi 24 février. 
d'une atroce plaie, et certains furent tués ${ }^{11}$; à Compiègne enfin, nombre des siens ont été frappés, occis et capturés ${ }^{12}$.

Cruauté est ici utilisée au sens propre: qui aime le cru, et donc le sanguinolent, c'est le fait des bêtes sauvages et non des êtres humains. Jeanne est irréductible à la condition ordinaire, être mi-humain mi-bestial, pour cette seule raison, elle méritait d'être soustraite à la compagnie de ceux qui, heureusement, ne sont donc pas entièrement ses semblables. Dans sa lettre à Cauchon, Henry VI rappelle qu'elle a commis de "cruels homicides $»^{13}$ et, de fait, c'est bien du sang humain dont elle est assoiffée, selon le promoteur : sanguinis humani crudeliter sitibunda ${ }^{14}$.

\section{L'accusation : verser le sang, une affaire d'hommes}

Cette cruauté qui fait bon marché de la souffrance d'autrui et de la sienne propre est en étroite correspondance avec d'autres crimes imputés à la Pucelle : se dire chef de guerre, porter les habits masculins, haïr la paix. Tout cela forme un ensemble qui, tout en restant dans le contexte de l'étrangeté et de la dangerosité religieuse de Jeanne - selon le point de vue des accusateurs, s'entend-, replace également le sang versé dans une tout autre perspective que celle du destin particulier de la Pucelle.

Ayant abandonné «l'habit de femmes, elle a imité le comportement de l'homme $»^{15}$, elle a pris «sans pudeur l'habit infâme et l'état des hommes ${ }^{16}$, elle a " assumé présomptueusement et orgueilleusement la domination sur des hommes ${ }^{17}$. La guerre, activité virile, est une affaire qui ne concerne pas les femmes. Ce n'est pas combattre qui est le véritable scandale aux yeux des juges ecclésiastiques: les Pères de l'Église ont depuis longtemps montré que la guerre n'est pas mauvaise en soi et les théologiens et juristes des $\mathrm{XIV}^{\mathrm{e}}$ et $\mathrm{XV}^{\mathrm{e}}$ siècles ont largement ajouté à leurs arguments ${ }^{18}$, après que saint Bernard a annoncé que la mort au combat était méritoire $^{19}$. Mais le scandale venait de l'usurpation de la condition masculine par une femme. Dans le cas précis et hors nature de Jeanne, la femme, par essence inapte au combat, ne peut se battre et faire combattre que par cruauté délibérée; elle rend alors, par cela seul, la guerre injuste et trompe ainsi ceux qu'elle entraîne ${ }^{20}$. À la

${ }^{11}$ Ibid., I, p. 268, session du 27 mars : de exercitu eius quamplures et ipsamet atroci vulnere sauciati fuerant et quidam interfecti.

${ }^{12}$ Ibid., t. I, p. 218, session du 27 mars : vulneratis, interfectis et captis multis de suis.

${ }^{13} \mathrm{Ibid}$., t. t. I, p. 12.

${ }^{14}$ Ibid., t. I, p. 192.

${ }^{15}$ Ibid., t. I, p. 16.

${ }^{16}$ Ibid., t. I, p. 191

${ }^{17} \mathrm{Ibid}$., t. I, p 263.

${ }^{18} \mathrm{P}$. Contamine, "L'idée de guerre à la fin du Moyen Âge. Aspects juridiques et éthiques », Comptes-rendus de l'Académie des Inscriptions et Belles-lettres, Paris, 1979, p. 73-74; et du même : «La théologie de la guerre à la fin du Moyen Âge : la guerre de Cent Ans fut-elle une guerre juste? », dans Jeanne d'Arc, une époque, un rayonnement. Colloque d'Orléans (1979), Paris, 1982, p. 9-21.

${ }^{19}$ Dictionnaire de théologie catholique, t. 6, col. 1912-1913.

${ }^{20}$ Selon saint Augustin (Contra Faustum, Patrologie lat., t. XLII, col. 447), la guerre injuste est faite par cupiditas, crudelitas, impacatus atque implacabilis animus. 
limite, le parti de Charles est excusable car, abusé, il a mal tourné ses vertus guerrières ; Jeanne en revanche est impardonnable.

Répandre le sang n'est, comme la guerre, pas répréhensible s'il s'agit d'actes masculins, cela le devient si une femme s'en mêle et se bat, car c'est évidemment pour tuer et non pour avancer la paix. Coupable d' " horribles homicides et détestables cruautés », Jeanne entraîne avec elle dans sa faute l'ensemble de son parti et pervertit la valeur offensive en avidité sanguinaire, qu'il est donc juste de dénoncer et d'anéantir.

\section{Les défenseurs de Jeanne : le silence officiel}

Les défenseurs de Jeanne d'Arc, promoteurs de la proclamation de la nullité de la sentence prononcée contre elle, sont extrêmement gênés pour répondre à l'accusation de cruauté, lancée lors du procès précédent. Il était impossible de nier que Jeanne ait combattu, que certains hommes d'armes de son parti aient laissé leur vie à Compiègne ou à Paris, qu'elle ait écrit sa lettre aux Anglais, que Glasdale et d'autres aient péri dans les eaux de la Loire, qu'elle ait laissé Franquet d'Arras aux mains du bailli de Senlis qui le fit décapiter. La guerre ne va pas sans verser du sang.

Pour les avocats de la famille de Jeanne et les serviteurs du roi, mieux valait passer rapidement sur ce qui semblait justement le plus gênant à l'époque et qui pour nous ne l'est plus : la liaison entre guerre, habits d'homme et accusation de cruauté. Il n'en est pas question dans les productions des demandeurs qui se contentent de dire que l'habit masculin que reprit la jeune fille dans sa prison était le plus propre à empêcher qu'on la viole ${ }^{21}$. On veille à ne pas aborder la question de la guerre et du sang versé. Les attendus de la sentence estiment que «les actions de la dite défunte sont plus dignes d'admiration que condamnables [...] En s'étonnant fort du jugement porté contre elle, en raison de sa forme et du fond; et en disant qu'il est très difficile de porter un jugement sûr en de telles questions ». La sentence elle-même affirme que les articles insérés dans la sentence de Rouen sont calomnieux, que l'abjuration fut obtenue par tromperie et que tout, procès, abjuration, sentence à mort et exécution est nul et sans valeur. Mais nous n'avons aucune déclaration particulière sur l'accusation de cruauté et de sang versé. Il faut aller les chercher auprès des consultations des sages, dans les traités inclus dans le procès en nullité et les paroles des témoins. Le résultat n'est pas sans intérêt.

\section{Les traités : la cruauté n'est pas là où les juges de Rouen la trouvaient}

Les huit traités qui accompagnent le procès en nullité dans l'édition de Pierre Duparc sont de longueur et de valeur inégale, et n'ont guère fait l'objet d'études poussées, largement, je le crains, en raison de l'absence de traduction ${ }^{22}$. La plupart, à

${ }^{21}$ Procès en nullité de la condamnation de Jeanne d'Arc, éd. P. Duparc, Paris, Klincksieck, 1979-1988, 5 vol. (Société de l'histoire de France), t. II, p. 24, par la suite : Procès en nullité. Je n'ai pas ajouté pour ce travail les textes publiés par Lanery d'Arc en 1889, ni le traité de Jacques Gélu, De adventu Johanne.

${ }^{22}$ Notons cependant sur Élie de Bourdeilles, l'article de J. M. Pinzino, « Speaking of angels : a fifteenth century bishop in defense of Joan of Arc's mystical voices ", dans Fresh verdicts on Joan of Arc, éd. B. Wheeler et C. T. Wood, New York, 1996, p. 161-176. 
propos de la guerre et de Jeanne, se contentent de reprendre de façon plus ou moins originale la question générale de la guerre juste pour expliquer la valeur positive, voire divine, du combat de Jeanne, ce qui n'est pas de notre propos ici, car ils ne répondent pas vraiment à l'accusation de cruauté particulière que manifesterait le sang versé par cette femme.

Élie de Bourdeilles, cependant, répondant méthodiquement aux accusations des juges de Rouen, est amené à traiter de la cruauté. Il élude magnifiquement l'aspect sanglant du cru en se rapportant aux Étymologies d'Isidore de Séville : crudus enim est asper et durus ${ }^{23}$. La cruauté est alors décharnée et toute morale ; elle se définit par son contraire : la clémence qui permet d'en référer à Sénèque. Jeanne est lavée de l'accusation de cruauté - sans que le mot sang soit écrit - grâce à la reprise des paroles mêmes de la Pucelle: ipsa assuerit se portasse vexillum, in quo erat scriptum "Jhesu Maria» pro evitandi ne interficeret aliquum ${ }^{24}$. L'étendard d'une main, le bouclier à l'autre bras, il était impossible de blesser quiconque. Des arguments à peu près identiques se retrouvent chez Martin Berruyer ${ }^{25}$.

Mais, à la différence des textes totalement officiels, les savants en arrivent à revenir sur le sang versé dans le royaume de France au temps de Jeanne : d'abord elle avait en horreur l'effusion de sang, rappellent à la fois Martin Berruyer ${ }^{26}$ et Jean de Montigny: maxime ab effusione sanguinis quantum est possibile abstinendo ${ }^{27}$. Robert Ciboule, assez embarrassé pour concilier une jeune fille et la guerre, suit un raisonnement qui excuse à la fois les guerriers et la femme : cette guerre était juste, car elle se portait au secours de la patrie et, là ou il y a justice, il n'y a pas de cruauté : quamvis non sine sanguinis humani fiat effusione. En portant son étendard au combat, Jeanne évitait de tuer, bien qu'il lui fût licite en ce cas de guerre juste de verser le sang ennemi ${ }^{28}$.

Martin Berruyer peut aller plus loin encore en retournant assez facilement la cruauté au compte des Anglais : la mort de Jeanne fut une des manifestations de leur cruauté, et non des moindres. Mais la promenade militaire du sacre, pendant laquelle les villes s'ouvrirent comme par miracle devant le roi et la Pucelle, avait ouvert l'ère de la reconquête presque sans victimes, et les cruels tourments causés par les Anglais prirent fin avec leur défaite. Le recouvrement de la Normandie et de l'Aquitaine se fit même absque pene sanguinis Gallorum effusione ${ }^{29}$, ce qui paraît bien évidemment une notation pour les besoins de la cause. La victoire annoncée par la Pucelle se devait d'être aussi économe du sang français que l'avait été l'héroïne de son vivant.

${ }^{23}$ Procès en nullité, t. II, p. 138-139.

${ }^{24}$ En réponse au $57^{\mathrm{e}}$ argument du libelle de Jean Estivet, Jeanne avait répondu : quod ipsamet portabat illud étendart cum intraret in adversarionis, pro evitando aliquem interficeret; et dixit quod numquam interficerat hominum.

${ }^{25}$ Procès en nullité, t. II, p. 241 : portabat vexillum suum ne aliquem interficeret.

${ }^{26}$ Ibid., t. II, p. 241 : aborreat efusionem sanguinis humani, quasi pia, lenis et clemens.

${ }^{27}$ Ibid., t. II, p. 284.

${ }^{28}$ Ibid., t. II, p. 357 : quamvis fuisset sibi licitum in bello justo sanguinemen fundere hostium, ad evitandum omnem crudelitatis notam, ipsa, ut in processu confessa es, portabat vexillum pro evitando ne ipsamet interficeret adversarios...

${ }^{29}$ Ibid., t. II, p. 234. 


\section{Femmes fortes de la Bible : les choix}

Autant que le retournement de la cruauté de Jeanne vers ses tourmenteurs, l'argumentation des théologiens et juristes vise finalement à trouver des équivalents bibliques et canoniques à son aventure. Je ne parlerai pas des femmes qui s'habillèrent en hommes pour mener une vie édifiante, de préférence monacale, ce n'est pas notre sujet - même si c'est un des points favoris de la gender history anglosaxonne ${ }^{30}-$, mais des modèles bibliques, ceux de Judith et Deborah, car ils amènent à revenir sur les manifestations militaires et sanglantes de la carrière de notre héroïne et, par contre coup, permettent de relire peut-être autrement les modèles proposés.

Judith est citée en miroir de Jeanne par tous les auteurs et par Bréhal dans sa récapitulation des arguments en faveur de la nullité du jugement de Rouen. Le modèle n'est pas sans danger, tout en étant bien loin en réalité de la Pucelle. Pour Gerson et Berruyer, leur trait commun est d'avoir été toutes deux suscitées par Dieu, ils ne veulent guère aller plus loin. Élie de Bourdeilles est fort ennuyé d'avoir à justifier la provocation sexuelle et la séduction physique opérées par l'héroïne d'Israël sur Holopherne et il insiste, afin de rapprocher la vierge Jeanne de la veuve de Manassé, sur le fait que toutes deux étaient menées par un ange et que ce dernier a empêché Holopherne de faire subir les derniers outrages à la belle Judith : et non permisit eam coinquinari, sed sine pollutione peccati revocavit eam ${ }^{31}$.

Bréhal justifie par l'inspiration divine deux démarches différentes pour un but similaire : Jeanne en vêtement d'hommes et Judith se parant avec soin n'ont en vue que le salut de la patrie. Il n'y a chez l'une ou chez l'autre aucune cruauté mais une impérieuse nécessité : «Fais seigneur, dit Judith, que sa propre épée abatte sa superbe»; et, poursuit Bréhal, la glose ajoute : «Elle ne dit pas cela par plaisir mais par amour de la justice».

Judith, meurtrière qui a usé de ses charmes pour le bien commun, demeure une référence féminine trouble mais conforme au rôle naturel des femmes qui est de séduire par les vêtements et les parfums, de tromper au creux des oreillers et de dissimuler la preuve de leur crime dans les courtines du lit, après avoir épuisé le mâle ${ }^{32}$. À dire vrai, Jeanne d'Arc est bien loin de coïncider avec le modèle !

Déborah est un exemple plus pertinent peut-être, dans la mesure où c'est elle, la prophétesse qui sur l'inspiration de Dieu convoque les armées d'Israël pour chasser les Cananéens et, à la demande de leur général Baraq, marche avec elles ${ }^{33}$. Cette femme aussi est citée par Gerson, Élie de Bourdeilles, Berruyer et Jean Bréhal. Mais Robert Ciboule est le seul à évoquer les événements qui suivirent la victoire de Baraq, l'étrange et sanglante aventure de Jehel, reprise dans le cantique de Déborah: Sisera, le général cananéen, vaincu et en fuite, arrive à la tente de Jehel la femme d'Haber :

${ }^{30} \mathrm{M}$. Warner, Joan of Arc. The image of female heroism, New York, 1981, et V. R. Hotchkiss, Clothes makes the man: female cross dressing in medieval religion, litterature and history, New York, 1996 : «Transvestim on trial : the case of Jeanne d'Arc», p. 49-68.

${ }^{31}$ Procès en nullité, t. II, p. 45.

${ }^{32}$ Judith, XIII, 1-10. Dans la Bible, l'épuisement d'Holopherne est celui de l'ivresse.

${ }^{33}$ Juges, IV, 4-10. 
Il demandait de l'eau, elle a donné du lait/ dans la coupe précieuse, elle a offert la crème./ Elle a tendu la main pour saisir le piquet,/ La droite, pour saisir le marteau./ Elle a frappé Sisera, elle lui a brisé la tête./ Elle lui a percé et fracassé la tempe./ À ses pieds, il s'est écroulé,/ Il est tombé, il s'est couché34.

Cet atroce meurtre au féminin est, à nouveau, un modèle qui ne convient guère à Jeanne qui n'a pas attendu au coin du feu que l'ennemi frappe à sa porte pour l'attirer comme un chat et le frapper comme un chien.

Les modèles bibliques, en dépit ou peut-être en raison de leur caractère obligatoire pour le genre littéraire des traités, se révèlent irréductibles à l'originalité de la carrière de Jeanne d'Arc et Robert Ciboule frise le ridicule en supposant, tout en s'abritant derrière l'autorité improbable d'Alexandre de Halès, que Déborah avait pu s'habiller en homme pour suivre les armées ${ }^{35}$. Les femmes fortes de la Bible, Judith et Yahel, ont versé le sang en usant largement de leurs armes féminines, ce ne fut aucunement le cas de Jeanne, dont on ne peut en outre prouver qu'elle ait fait couler elle-même une goutte de sang. Le mieux était donc de laisser retomber la chape du silence sur une Pucelle assoiffée de cruauté.

\section{Les témoins de la défense : le cœur accusateur}

Les témoins convoqués pour soutenir les efforts d'annulation du procès de Jeanne ont eu à répondre à un certain nombre de questions précises. Comme toujours en matière de procès, leurs propos sont guidés par le questionnaire, il ne s'agit pas d'une enquête d'historiens ou de sociologue, mais en quelque sorte d'une contre-inquisistion. Il était, en réalité, bien difficile de prétendre que personne n'avait perdu la vie en conséquence directe des actions de l'hérö̈ne. Elle avait bel et bien mené ou accompagné des soldats décidés à en découdre de façon sanglante avec les Anglais. Même si Jeanne avait toujours tenté d'abord une négociation de paix, si elle avait laissé partir à Orléans et Beaugency les ennemis sans les poursuivre, si elle avait pleuré sur les blessés et les morts, il y avait eu des cadavres sur des champs de bataille, Glasdale s'était noyé en Loire. Aussi, ne demanda-t-on pas aux témoins leur avis sur l'accusation de cruauté. C'est indirectement qu'ils furent amenés à évoquer le sang des blessures que l'on donne et qu'on reçoit.

Jeanne avait peur du sang. Jean d'Aulon rapporte qu'elle n'en supportait pas la vue $\mathrm{e}^{36}$. Inversant, sans qu'on les en prie ouvertement, l'accusation de cruauté, Frère Pasquerel souligne que c'est son sang à elle qu'a versé la Pucelle : «demain le sang coulera au-dessous de mon sein ». Maître Jean Tiphaine rapporte que lorsque Jeanne malade dut être saignée, les Anglais manifestèrent une méchanceté sans pitié. N'était-ce pas cruauté que Warwick dise publiquement: "gardez vous de la saigner car elle est rusée et pourrait se faire mourir ", c'est-à-dire se laisser saigner à mort, puisque, selon ses accusateurs, elle était suicidaire ? Or Jeanne fut saignée et guérit, c'est le contrepoint de la notation de Jean d'Aulon selon laquelle « elle ne connais-

${ }^{34}$ Juges, V, 25-27.

${ }^{35}$ Procès en nullité, t. II, p. 377 : Et presumitur, ut expresse dicit magister Alexander de Halis, In secundum librum, quod Debora utabatur veste viril et armis militaribus ad expugnandos inimicus populi Israel.

${ }^{36}$ Procès en nullité, t. I, p. 392. 
sait pas les maladies des femmes». L'horreur de verser le sang alla-t-elle réellement jusqu'à l'aménorrhée ? Le propos n'est pas ici de la pathologie éventuelle du phénomène Jeanne $\mathrm{d}^{\prime} \mathrm{Arc}^{37}$, mais il me semble que, sans abuser du terme, la symbolique affleure dans cette remarque incidente d'un témoin privilégié.

La Pucelle a donc horreur du sang qui coule et encore plus quand il vient des Français, c'est ce que veulent montrer Louis de Coûtes et frère Pasquerel. Le page eut son baptême du feu, si l'on ose dire, à la bastille Saint-Loup ${ }^{38}$. Quand Jeanne apprit que l'on s'y battait, elle aurait dit, selon son confesseur, : «Le sang de nos gens coule sur la terre $»^{39}$. Le page se souviendra toujours de l'algarade: «Vous ne me diriez pas que le sang de France fût répandu ! ${\aleph^{40}}^{0}$ Outre que, de l'un à l'autre témoin, le sang des hommes devient celui du royaume, et peut-être de la patrie, l'accusation des Anglais affirmant la cruauté de Jeanne se trouve anéantie: c'est hors de sa présence et sans son consentement, et non par son insatiable cruauté, que fut versé le premier sang de son aventure. L'étrange qualificatif qu'elle lance à son page, "Ah sanglant garçon... »" inverse, volontairement n'en doutons pas, et cela avec une grande finesse, les allégations anglo-cléricales de l'accusation. Louis de Coûtes devient, pour avoir laissé dormir la Pucelle, le sacrificateur sanglant des gens de son parti et il innocente ainsi une Jeanne qui arrive trop tard pour proposer la paix avant le combat ${ }^{42}$. À Saint-Loup, en mai 1429, le sang a coulé avant qu'elle n'ait pu l'empêcher de se répandre, le processus de la victoire guerrière avec son cortège de violences est enclenché. Dieu a choisi à sa place.

$\mathrm{Au}$ cœur des combats, aux prises avec la haine de ses ennemis anglobourguignons, Jeanne est un cœur pur. Nous le lisons aujourd'hui, ce qui n'était certes pas prévu, dans la plupart des réponses que nous livre le procès de Rouen. Un des témoins des enquêtes de 1450 et 1452 le prouve, ou tente de le prouver, par l'intrusion de l'impossible, voire du miracle, et ce qui ne manque pas de sel, il fut aussi un des assistants du procès de Rouen. Maître Jean Massieu, repenti sincère peut-être - laissons-lui le bénéfice du doute -, conte, certes par ouï-dire, que sur la place du Vieux-Marché, au soir du supplice de Jeanne, «une fois son corps brûlé et réduit en cendres, son cœur resta intact et plein de sang $»^{43}$.

Le cœur de Jeanne, lieu de sa vérité profonde dont Dieu avait connaissance ${ }^{44}$, siège de son courage, de sa vaillance et aussi de son anima, car selon Thomas d'Aquin, l'âme est dans le sang, était si peu cruel qu'il garda intact le sang pur de l'héroïne. Après le sacrifice, le cœur révélateur de 1431 prouvait l'inanité de l'accusation de cruauté et au contraire reportait sur les accusateurs et les tortionnaires la honte du

${ }^{37} \mathrm{O}$. Bouzy, «Le dossier médical », dans Jeanne d'Arc, une passion française, L'Histoire, $\mathrm{n}^{\circ} 210$, mai 1997, p. 58-59.

${ }^{38}$ Procès en nullité, t. I, p. 363.

${ }^{39} \mathrm{Ibid}$., t. I, p. 392.

${ }^{40} \mathrm{Ibid}$., t. I, p. 363.

${ }^{41} I d$.

${ }^{42}$ On notera accessoirement que le sang anglais est passé sous silence alors que, pour les témoins, la guerre étrangère est désormais finie.

${ }^{43}$ Procès en nullité, t. I, p. 347.

${ }^{44}$ «Les vues de Dieu ne sont pas comme les vues de l'homme, car l'homme regarde à

l'apparence, mais Yahvé regarde au cœur» (I Samuel, XVI, 7). 
sang innocent, devenu ici déjà celui d'une martyre. «Le sang immaculé d'une innocente crie certainement devant le trône du Seigneur», proclamaient les motifs de droit du Procès en nullité. Les rédacteurs de la procédure de 1456 nous prouvent aujourd'hui que, tout sincères qu'ils fussent, ils étaient également de fins rhétoriqueurs. 